\title{
Atom-probe Tomography, Small Angle Neutron Scattering, Transmission Electron Microscopy, Positron Annihilation Spectroscopy and X-ray Absorption Spectroscopy Characterization of Nano-scale Features in Nanostructured Ferritic Alloys
}

\author{
G. R. Odette*, N. J. Cunningham*, E. A. Marquis**, S. Lozano-Perez**, V. de Castro**, P. \\ Hosemann $* * *, * * * *$, E. Stergar****, S. Liu***** and C.U. Segre***** \\ * Materials Department, University of California, Santa Barbara, CA \\ ** Department of Materials, University of Oxford, Parks Road, OX1 3PH Oxford, UK \\ *** Materials Science and Technology Division, Los Alamos National Laboratory, Los Alamos NM \\ **** Department of Physical Metallurgy, University of Leoben, Loeben, Austria \\ ***** Physics Department, Illinois Institute of Technology, Chicago, IL
}

Nano-dispersion strengthened $14 \mathrm{Cr}$ ferritic alloys (NFAs), with small concentrations of Y, Ti and excess $\mathrm{O}$, contain a high density of thermally stable Y-Ti-O enriched nano-scale features (NFs) which provide both high creep strength and irradiation damage resistance. Mechanical alloying by ball milling dissolves $\mathrm{Y}_{2} \mathrm{O}_{3}$ in Fe alloy powders and the $\mathrm{Y}$ and $\mathrm{O}$ then precipitate along with Ti to form NFs during hot consolidation. The NFs have been studied by small angle neutron scattering (SANS), three-dimensional atom probe tomography (APT) (Fig.1a), various types of transmission electron microscopy (TEM), positron annihilation spectroscopy (PAS) and X-ray absorption spectroscopy (XAS). However, the compositions and structures of various NFs are still not yet well understood. The NFs appear to range from coherent solute enriched GP-type zones, or incoherent sub-oxides (in APT studies), to stoichometric complex oxides $\left(\mathrm{Y}_{2} \mathrm{TiO}_{5}\right.$ and $\left.\mathrm{Y}_{2} \mathrm{Ti}_{2} \mathrm{O}_{7}\right)$ as reported in some SANS and TEM studies. We cross compare the results from the various techniques both for a single alloy MA957 as well as for several other similar NFAs.

The various techniques are generally in good agreement on the sizes, number densities and volume fractions of the NFs. However, APT studies show high $(\mathrm{Ti}+\mathrm{Y}) / \mathrm{O}$ and $\mathrm{Ti} / \mathrm{Y}$ ratios that are inconsistent with complex oxides, as well as with some TEM and SANS results. The APT element maps indicate complex NF structures are composed of Y-T-O enriched cores surrounded by TiO rich shells (Fig. 1b). The $\mathrm{Ti} / \mathrm{Y}$ ratios decrease in larger $\mathrm{NFs}$, but high $(\mathrm{Ti}+\mathrm{Y}) / \mathrm{O}$ ratios persist even for features that are presumably in the size-range of well-developed complex oxides. SANS is generally consistent with a mix of various complex oxides in MA957, but not with the high Ti/Y ratios unless the NFs contain considerable quantities of Fe. Preliminary XAS data appears to be consistent with oxides including a defected TiO phase. Phase contrast TEM images NFs down to small sizes. The diffracting features that have been observed in NFAs, at slightly larger sizes, have been identified as complex oxides. Energy filtered TEM (EFTEM) on larger features has been shown to be consistent with complex oxides in some NFAs. EFTM also images smaller Y-Ti-O enriched NFs, but their compositions have yet to be quantified.

Limitations of the various techniques are discussed and conclusions as to the nature of the various NFs are summarized Additional experiments will be carried out with special emphasis on characterizing the larger features using a variety on TEM-based methods. 
[1] GRO and NJC acknowledge the support of the US DOE Office of Fusion Energy Sciences and Nuclear Energy Program; EAM acknowledges the support of EPSRC and the Royal Society, S. Lazono Perez acknowledges the support of University of Oxford Department of Materials; VdC acknowledges the support of FP6 Euratom Research and Training Programme on Nuclear Energy; P Hosemann acknowledges the support of US DOE Nuclear Energy Program and the University of Leoben; E. Stergar acknowledges the support of the University of Leoben; S. Liu and C.U. Segre acknowledge the support of the US DOE Nuclear Energy Program. The SANS studies were carried out at the NIST Center for Neutron Research.
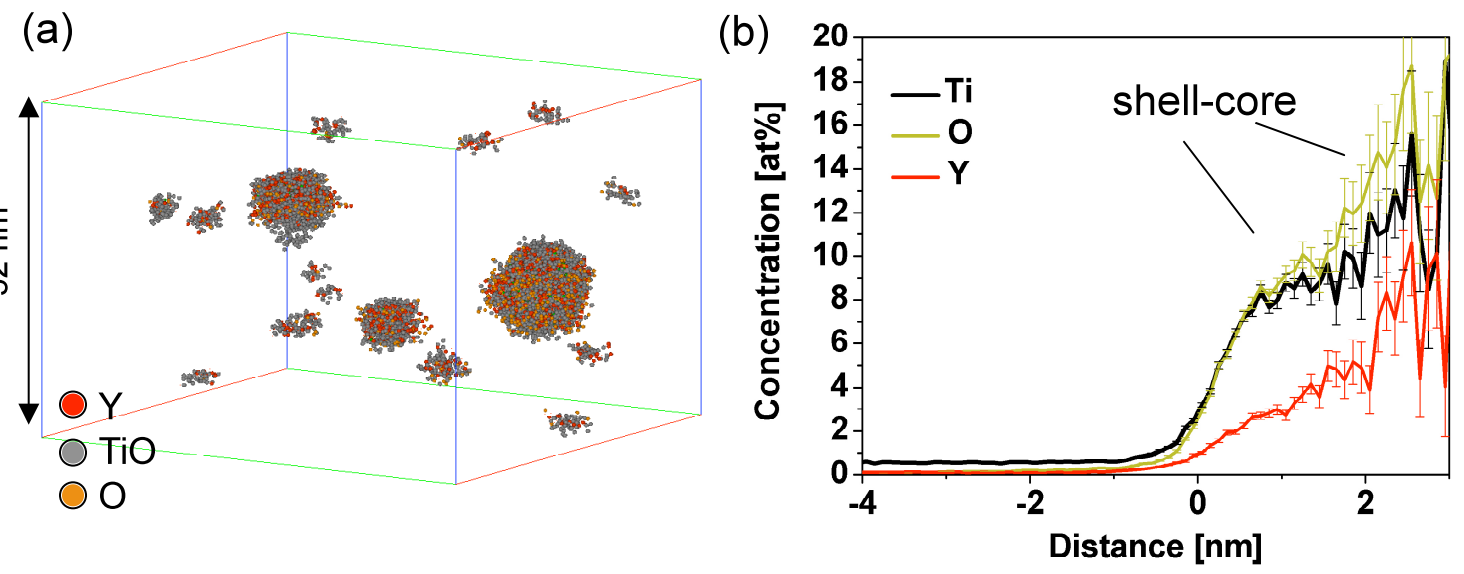

Figure 1: (a) three-dimensional reconstruction from atom-probe datasets showing the Ti-Y-O rich features in sizes from $\sim 1-20 \mathrm{~nm}$. (b) Proximity histogram showing the core-shell structures of the nanoscale features. 